\title{
Inspección mediante técnicas no destructivas de un edificio histórico: oratorio San Felipe Neri (Cádiz)
}

\author{
Inspección with non destructive techniques of a historic \\ building: oratorio San Felipe Neri (Cádiz)
}

C. Rodríguez Liñán ${ }^{(*)}, M^{a}$ J. Morales Conde ${ }^{(*)}$, P. Rubio de Hita ${ }^{(*)}$, F. Pérez Gálvez ${ }^{(*)}$

\section{RESUMEN}

El objetivo de este artículo es dar a conocer los resultados obtenidos al aplicar técnicas no destructivas de inspección (ultrasonidos y termografía) durante las obras de reparación de un edificio de gran valor histórico y artístico.

Estas técnicas no destructivas se han aplicado en la inspección de la cubierta de madera del edificio para detectar distintos estados de deterioro, pérdidas de densidad y defectos, con el objetivo de evaluar su estado de conservación. Se ha realizado un trabajo de campo donde se ha aplicado ambas técnicas conjuntamente, los ultrasonidos, técnica eficaz para establecer el diagnóstico de una estructura de madera, y la termografía, técnica menos experimentada en la inspección in situ de este material. El objetivo es acotar el alcance y las posibilidades reales de utilización de cada una de las técnicas. En este sentido, se aplica la metodología de ultrasonidos desarrollada y publicada por las autoras y se estudia la termografía como herramienta de inspección in situ analizando las dificultades añadidas y parámetros de interferencia no detectados en laboratorio.

Las conclusiones del trabajo ponen de manifiesto que la unión de la técnica de ultrasonidos y la termografía constituyen una buena herramienta para la inspección in situ de las estructuras de madera y para evaluar sus condiciones permitiendo establecer un diagnóstico adecuado. La termografía permite detectar distintos contenidos de humedad y distintos materiales $y$, por otro lado, los ultrasonidos los distintos grados de deterioro o pérdida de densidad en zonas localizadas con elevado contenido de humedad de las piezas.

464-8

Palabras clave: Diagnosis, inspección, termografía, estructuras de madera, ultrasonidos

\section{SUMMARY}

The main aim of this article is showing the results that have been obtained when non destructive techniques (ultrasound and thermography) are applied to a building with an important artistic and historical value.

These non-destructive techniques have been applied in the inspection of the wooden roof structure to detect different states of deterioration, loss of density and defects. The aim is the assessment the state of the preservation of the wooden structure. A fieldwork has been carried out, where the two techniques have been applied together, ultrasound, an effective technique to establish the diagnosis of a wooden structure, and the thermography, a technique less tested in the in situ inspection of this material. The aim is checking the range and the real possibilities of using of each technique. In this sense, the methodology of ultrasound developed and published by the authors is applied and the thermography like in situ inspection tool, analysing additional difficulties and interference parameters not found in laboratory.

The conclusions of this study show that the union of the ultrasound technique and the thermography is a good tool for on-site inspection of wooden structures and to assess their conditions allowing establishing a proper diagnosis. Thermography can detect different moisture contents and different materials and, on the other hand, ultrasound detects different states of deterioration or loss of density in specific areas with a high moisture content of the pieces.

Keywords: Diagnosis, inspection, thermography, wooden structures, ultrasound 


\section{INTRODUCCIÓN}

Cuando nos enfrentamos a un proyecto de rehabilitación o restauración arquitectónica de un edificio existente surge la necesidad de contar con herramientas y métodos que nos permitan tener un conocimiento objetivo de la realidad del edificio y de su estado de conservación. El desconocimiento o la falta de información nos pueden conducir a establecer un diagnóstico erróneo y desembocar en un proyecto de actuación poco acertado. Métodos de inspección desarrollados hasta ahora utilizando la técnica de ultrasonidos (1) nos permiten evaluar el grado de deterioro o pudrición de una estructura de madera y nos ayudan a tomar decisiones sobre la necesidad de reparar o sustituir un determinado elemento constructivo. Un requisito indispensable para la aplicación de este método es tener que establecer un contacto directo con la superficie del elemento a inspeccionar que nos permita efectuar la medición. La inaccesibilidad o la dificultad que implica acceder a ciertos espacios y estructuras conllevan a que, en estas ocasiones, la operatividad del método se vea cuestionada. Es por ello que surge la necesidad de buscar un método alternativo que nos permita poder analizar aquellos espacios y estructuras que puedan presentar un problema de accesibilidad. En este sentido la termografía se perfila, a priori, como una herramienta de inspección no destructiva que sin necesidad de establecer un contacto directo permite obtener resultados sobre grandes áreas y con una sola medición

\section{LA TERMOGRAFÍA DE INFRARROJOS Y LOS ULTRASONIDOS}

La termografía es una técnica que, basada en los principios físicos de transmisión de calor, permite obtener a partir de la energía emitida por un objeto, en el rango de infrarrojos, la temperatura superficial del mismo (2). Esta radiación es recogida por una cámara termográfica, a través de unos dispositivos ópticos, y transformada en señales eléctricas. De esta forma se obtienen los Ilamados termogramas, mapas térmicos donde cada tono de color representa la temperatura de la superficie en ese punto estableciéndose según un rango de valores las diferencias de temperaturas del objeto. Esta radiación, definida físicamente mediante la ley de Stefan-Boltzmann (3), es proporcional a la temperatura del cuerpo y a las propiedades térmicas propias del material como la emisividad, que a su vez es función de la longitud de onda, de la temperatura y del ángulo de incidencia. Así, objetos que tengan la misma temperatura superficial

pueden aparecer con distintas temperaturas si tienen distintas emisividades.

$$
\begin{array}{ll}
Q_{\text {emitida }}=\varepsilon \cdot \sigma \cdot A_{s} \cdot T_{s}^{4} \quad \mathrm{~W} \text { (vatios) } \\
\mathrm{Q}_{\text {emitida }} & \begin{array}{l}
\text { Componente emitida de la radiación } \\
\text { total que fluye desde el cuerpo. }
\end{array} \\
\varepsilon & \text { Emisividad del material. } \\
\sigma & 5,67 \times 10^{-8} \mathrm{~W} / \mathrm{m}^{2} \cdot \mathrm{K}^{4} \text { es la constan- } \\
& \text { te de Stefan-Boltzmann. } \\
A_{s} & \text { Área de la superficie. } \\
T_{s} & \text { Temperatura de la superficie. }
\end{array}
$$

Los cuerpos reales tienen una emisividad inferior a la unidad y, la radiación que fluye desde su superficie es la suma de tres componentes: la componente emitida (Qe), la reflejada desde el entorno $(\mathrm{Q} r)$ y la transmitida a través de ella (Qt). Dado que muchos cuerpos son opacos a la radiación infrarroja, la radiación resultante será la suma de la componente emitida y la reflejada.

$$
\mathrm{Q}_{\mathrm{sal}}=\mathrm{Q}_{\mathrm{em}}+\mathrm{Q}_{\mathrm{ref}}
$$

$\mathrm{Q}_{\text {sal }} \quad$ Radiación total que fluye desde la superficie de un cuerpo.

$\mathrm{Q}_{\mathrm{em}} \quad$ Componente emitida de la radiación total.

$\mathrm{Q}_{\text {ref }} \quad$ Componente reflejada de la radiación total.

Siendo:

$$
1=\varepsilon+\rho
$$

$\varepsilon$

$\rho$

En el caso de la madera, su emisividad toma un valor aproximado de 0,9 por lo que el $90 \%$ de la energía que fluye desde su superficie será energía emitida y, un pequeño porcentaje será reflejada desde el ambiente. Esto conlleva a que los resultados obtenidos a partir de una medición termográfica sean fiables.

Análogamente, nos encontramos que toda medición termográfica está condicionada por una serie de parámetros ambientales externos (temperatura ambiental, transmisión de la atmósfera, velocidad del viento,...), que pueden enmascarar los resultados obtenidos (4).

Por otro lado, se denomina ultrasonidos a la aplicación de una vibración de las partículas, cuya frecuencia es superior a los 20 $\mathrm{KHz}$, superior al límite de escucha humana. El margen superior de estas frecuencias es muy elevado pudiendo llegar a los $10^{9} \mathrm{~Hz}$. 
Su propagación en los diferentes medios materiales es análoga a la propagación de las ondas sonoras dentro del margen audible, aunque con una absorción mucho mayor, por lo que el aire se puede considerar prácticamente opaco para los ultrasonidos.

Para la verificación de materiales mediante ultrasonidos pueden emplearse dos técnicas:

Método de intensidad o de transmisión. En él un generador de alta frecuencia excita a un cristal piezoeléctrico que transmite una onda sonora a la pieza a verificar. Un segundo cristal colocado de manera opuesta al primero recibe parte de la onda de presión emitida y, mediante el efecto piezoeléctrico inverso, se produce un voltaje eléctrico que se amplifica y se lee su intensidad mediante un instrumento de medida.

Método del impulso eco. Con el método de impulso eco sólo se necesita un palpador, es decir, el cristal es emisor y receptor a la vez en un mismo palpador. En este método, como en el anterior, se mide la presión sonora sólo que en este caso se mide la presión sonora reflejada y no la transmitida.

La elección de uno u otro método dependerá de la microestructura del material a verificar.

Para la verificación de materiales homogéneos el impulso eco es más adecuado. En estos materiales la velocidad de propagación del sonido puede considerarse constante $y$, se puede localizar la posición exacta de una discontinuidad así como el tamaño de la misma. No obstante, para materiales heterogéneos, como la madera, es más adecuado utilizar el método de transmisión ya que si se emplea el método del impulso eco, la energía de los impulsos ultrasónicos queda reflejada en las superficies límites y en los poros, produciéndose una dispersión del impulso en todos los sentidos en el interior del materia, I por lo que la energía reflejada es muy pequeña en relación con la emitida. Por ello, lo más adecuado es utilizar dos palpadores, uno emisor y otro receptor.

\section{LA TERMOGRAFÍA DE INFRARROJOS APLICADA A LA MADERA}

Las propiedades térmicas de la madera que intervienen en una inspección con termografía son el calor específico, la conductividad térmica y la difusividad térmica (5).

El calor específico corresponde a la cantidad de calor necesario para incrementar un grado de temperatura de un material por unidad de masa $\left(\mathrm{J} / \mathrm{Kg}^{\circ} \mathrm{C}\right)$. En la madera el calor específico es independiente de la especie de madera y de la densidad, pero varía con el contenido de humedad $(5,6)$.
La conductividad térmica, cantidad de calor que pasa a través de la unidad de superficie para un cuerpo de una unidad de espesor en una unidad de tiempo $\left(\mathrm{W} / \mathrm{m}^{\circ} \mathrm{C}\right)$. La madera es un mal conductor del calor y tiene un valor bajo de conductividad térmica $\left(0,17 \mathrm{~W} / \mathrm{m}^{\circ} \mathrm{C}\right)$. Esto es así porque presenta una estructura porosa y, además está afectada por numerosos parámetros como la densidad, el contenido de humedad, las irregularidades estructurales y la temperatura $(5,6)$.

La difusividad térmica $\left(\mathrm{m}^{2} / \mathrm{s}\right)$ es obtenida de los parámetros anteriores y representa cuán rápido se difunde el calor por un material. La madera tiene un valor de difusividad térmica bajo $0,13 \times 10^{-6}$, por lo que significa que, en su mayor parte el calor es absorbido por el material y una pequeña cantidad de ese calor será conducido. Varía con la densidad y con el contenido de humedad $(3,5,6)$.

Con todo ello podemos interpretar que, a priori, es una técnica que nos permite detectar maderas con distintas densidades y zonas con distinto contenido de humedad y densidad.

Basado en estos principios varios investigadores han estudiado la posibilidad de detectar defectos en la madera y distintos estados de deterioro. Es el caso de Maldague (5) o Tanaka (6). Maldague ha realizado varios estudios para tratar de determinar, por un lado, el grado de pudrición de los postes de madera utilizados en el tendido eléctrico $y$, en una segunda fase pretende establecer una sistemática para poder definir límites para la detección de defectos en la madera, trabajo realizado también por Tanaka.

\section{LA TÉCNICA DE ULTRASONIDOS APLICADA A LA MADERA}

Para la aplicación de la técnica de ultrasonidos se han realizado ensayos no destructivos de determinaciones de contenido de humedad y medidas de tiempos de transmisión de ondas ultrasónicas, mediante la metodología desarrollada por Rodríguez-Liñán C y Rubio De Hita, P. (7). Esta metodología permite diagnosticar el estado de la madera, obteniendo una estimación del grado de deterioro de la misma, y evaluar la capacidad portante de los forjados y cubiertas en obras de rehabilitación.

\section{EQUIPO Y MATERIAL UTILIZADOS}

Para realizar los ensayos de termografía se ha empleado una cámara de la marca FLIR que trabaja en la banda espectral de 7,5 a $13 \mu \mathrm{m}$ (onda larga) y tiene una sensibilidad térmica de $0,10^{\circ} \mathrm{Ca} 30^{\circ} \mathrm{C}$ con una precisión de $\pm 2{ }^{\circ} \mathrm{C}, \pm 2 \%$. El tipo enfoque es manual 

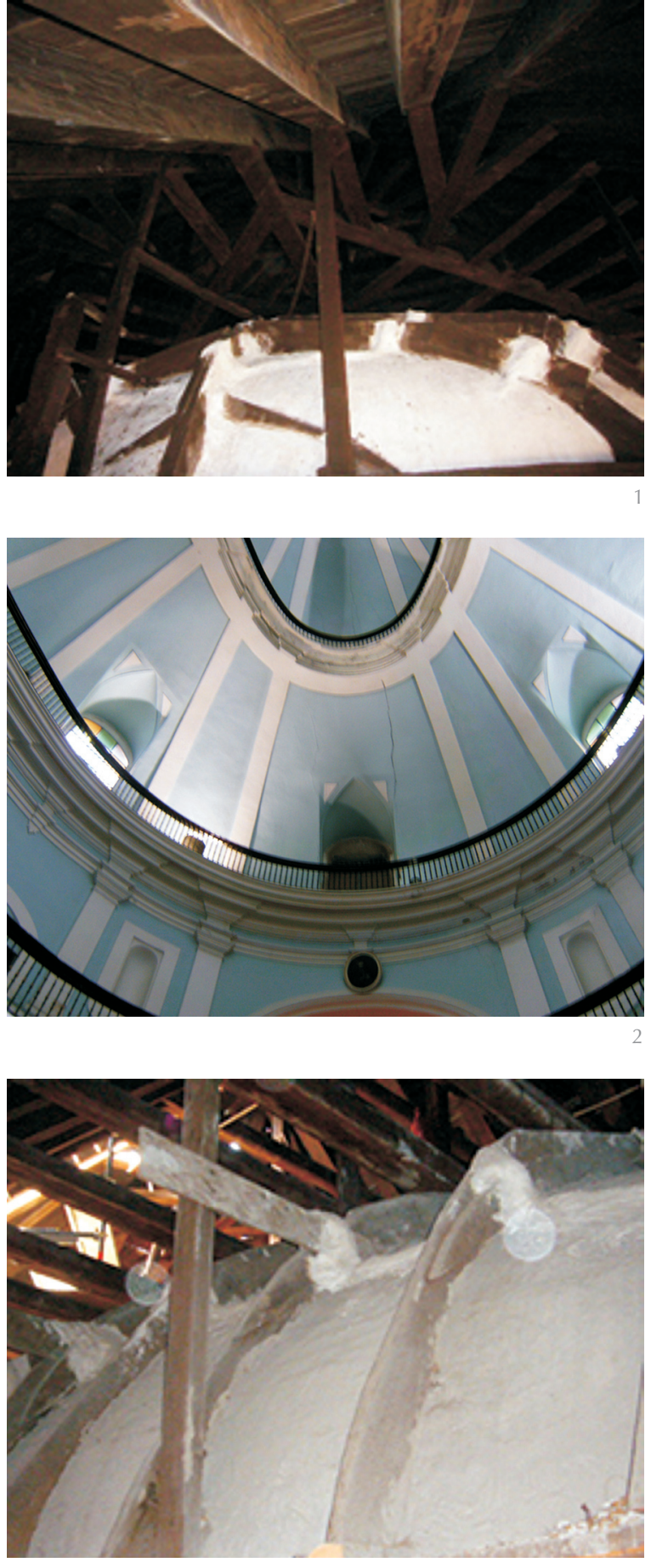

y tiene un campo de visión $23^{\circ}$ x $17^{\circ}$ y una distancia mínima de enfoque de 0,3 m. El tipo de detector es un microbolómetro no refrigerado (FPA) y su resolución es de $320 \times 240$ píxeles.

Para los ensayos de ultrasonidos se ha utilizado un aparato de ultrasonidos modelo BP $V$ de la marca Krautkramer. Este aparato nos da la lectura del tiempo de retardo en que la onda sale del palpador emisor hasta que es recibido por el palpador receptor, para lo que posee una pantalla de cristal líquido de cinco dígitos, en la que se muestra la lectura de tiempo en microsegundos. La precisión es de $\pm 0,1 \mu \mathrm{s}$. Para las mediciones se han utilizado unos palpadores cónicos ya que son de más fácil acoplamiento. La frecuencia de emisión de estos palpadores es de $50 \mathrm{KHz}$.

\section{EL EDIFICIO (ORATORIO DE SAN FELIPE NERI, CÁDIZ)}

El actual oratorio se inauguró el 17 de noviembre de 1719 y su construcción es el resultado de dos etapas (Figura 1). La primera se inició en 1688 en planos del alarife Blas Díaz y se terminó en 1717. Tras los estragos del terremoto y maremoto de 1 de noviembre de 1755, el templo se arruinó. En la segunda etapa, el maestro alarife Pedro Gutiérrez de San Martín (más conocido como maestro Afanador), construyó una nueva cúpula en 1764. Entre las reformas llevadas a cabo con posterioridad, hay que destacar la de 1775 que afecta a aspectos fundamentalmente decorativos $(8,9)$.

San Fernando y Cádiz fueron escenario de las Cortes Generales y extraordinarias (1810-1813). Las sesiones dentro del Oratorio tuvieron lugar desde 1811 hasta 1813, promulgándose en este templo la Constitución de 1812. En 1823 se celebraron las Cortes que acabó con el Trienio Liberal. La cripta del templo alberga el panteón de los diputados doceañistas y una urna las víctimas de la sublevación militar de marzo de $1820(8,9)$.

Próximamente, en el año 2012, se celebrará el segundo centenario de la Constitución y de las Cortes. Como consecuencia de estos actos, desde el arzobispado de Cádiz se están llevando a cabo obras de rehabilitación cuyo proyecto y dirección de obras están en manos del arquitecto D. Francisco Torres.

\subsection{Descripción del edificio}

El modelo del templo, inspirado en la Iglesia de San Jácome del Corso de Roma y siguiendo los modelos de iglesias jesuíticas, es de planta elíptica (sus ejes miden 26, 62 y $16,70 \mathrm{~m}$ ) sobre el que se abren siete capillas de planta rectangular.

Los muros tienen pilastras pareadas que recorren los diferentes cuerpos, superponiéndose las de orden jónico y corintio, con hornacinas en los intercolumnios, y entre las que se abren vanos que restan gravidez a la estructura. Es frecuente en las iglesias conventuales que las tribunas se asomen a la nave; aquí se sustituyen por una superposición de triple balconada sobre cornisas. La cúpula (Figura 2) es una gran estructura encamonada (Figura 3) de doble casquete decorada con 
fajas, que prolongan el ritmo de las pilastras inferiores y entre las que se abren vanos que restan gravidez a la estructura.

\subsection{Estado actual. Descripción de lesiones}

El principal problema que aparece en el Oratorio es de origen estructural y está provocado por el empuje inducido por la componente horizontal de la estructura de cubierta. Este empuje ocasiona la pérdida de trabazón de los muros perimetrales con la consiguiente aparición de grietas en los vértices de unión de dichos muros.

En el interior del Oratorio, dado que la cubierta tiene una doble estructura, el empuje de la cubierta exterior desplazada hacia el tambor se materializa a través de grietas que aparecen en los ejes principales de las elipses (Figura 4) de las cúpulas tanto en la cúpula inferior sobre la que apoya así como en la superior.

Observamos, en la visita realizada, que este problema había aparecido en el edificio con anterioridad puesto que se pueden distinguir los refuerzos realizados en las esquinas del tambor mediante perfiles metálicos.

Por otro lado, a partir de una inspección visual, observamos que el edificio presenta serios problemas de filtraciones. Estas filtraciones han ocasionado la pudrición de la madera de la estructura de cubierta, tanto de la tablazón como de sus pares (Figura 5) donde, además, existe un ataque superficial de carcoma común. No se ha detectado ataque de termitas en los pares aunque sí se ha podido localizar su presencia en algún otro elemento de madera del edificio como las carpinterías.

\subsection{La estructura de madera}

La cubierta del edificio está formada por una bóveda encamonada (Figura 3) que se cubre con una estructura de madera a ocho aguas (Figura 6). La estructura de madera (pares y nudillos) está realizada por vigas de madera aserrada de pino silvestre/laricio con una escuadría de los pares principales entre $20 x$ $25 \mathrm{~cm}$ y 14 x $23 \mathrm{~cm}$ y de $8 \mathrm{~m}$ de longitud. De esta estructura cuelga la estructura auxiliar de madera de sostén de la bóveda de yeso. El paño de la cubierta está formado por tablazón de madera sobre el que existe una capa de mortero de hormigón con restos de impermeabilizantes asfálticos resultado de anteriores intervenciones y una cubierta de teja.

\section{METODOLOGÍA}

La aplicación de la metodología de ultrasonidos desarrollada por este grupo permite obtener una estimación del grado de deterioro de la madera estudiada, pino silvestre y laricio. Se utilizan las correlaciones obtenidas, a partir de ensayos en laboratorio sobre 40 probetas del mismo tipo de madera, entre las medidas de velocidad de transmisión de ultrasonidos perpendiculares a la fibra y la pérdida de densidad y resistencia del material. Esta metodología también permite obtener una estimación del valor de resis-
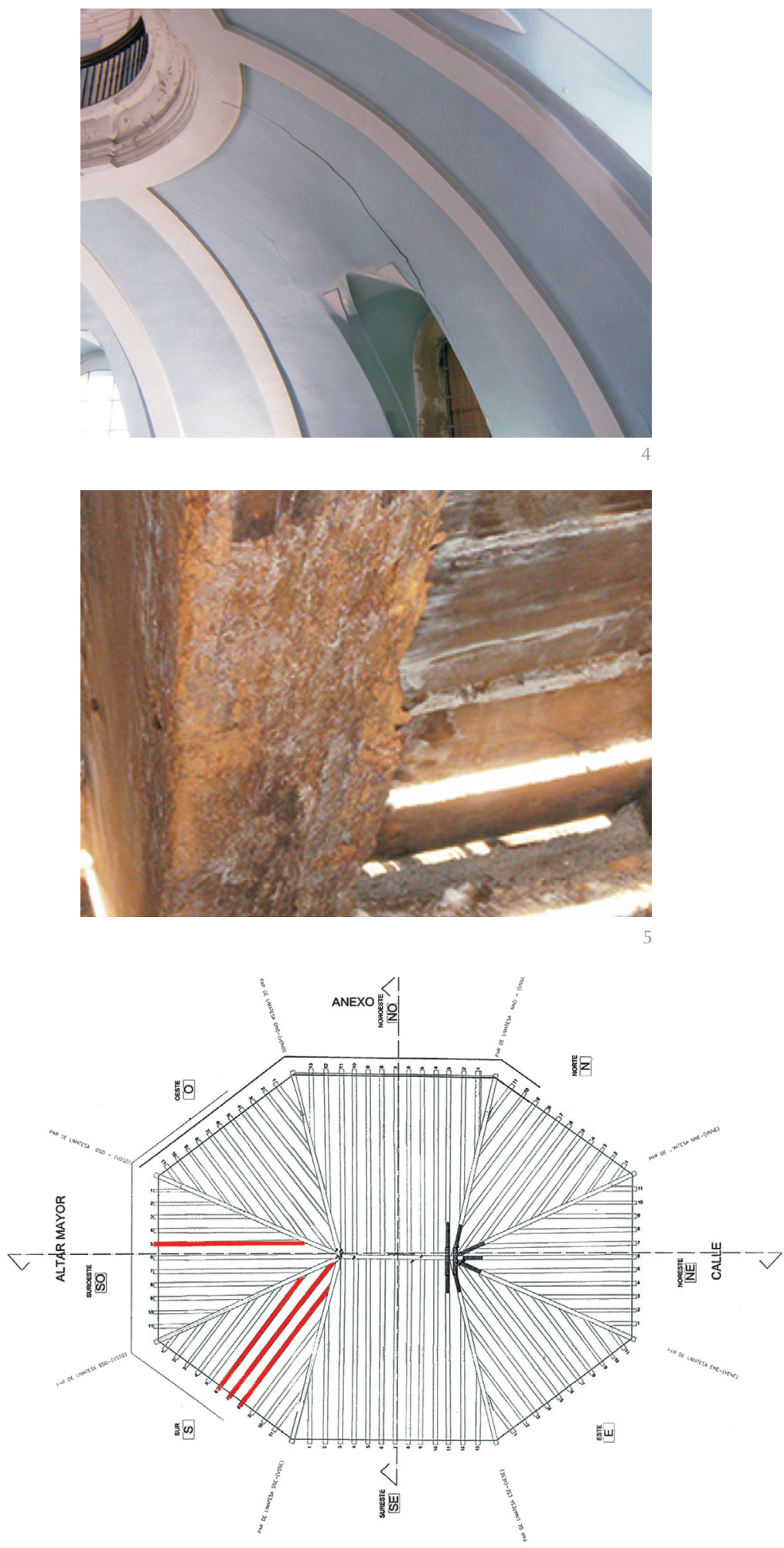
tencia a flexión de la madera, a partir de las velocidades de transmisión de ultrasonidos en sentido longitudinal a la probeta y su correlación con valores de tensión de rotura. Asimismo, dada la influencia de la humedad en los tiempos de velocidad ultrasónica se han desarrollado una serie de ecuaciones para referir todas las mediciones efectuadas a un mismo contenido de humedad de forma que los resultados de los diferentes elementos y secciones puedan ser comparados entre sí independientemente del contenido de humedad que estos presenten in situ.

- Estimación del deterioro de la madera:

A partir de las correlaciones obtenidas en ensayos realizados en laboratorio sobre muestras de madera de pino silvestre y laricio con distinto grado de deterioro asociando a unos valores de pérdida de densidad y resistencia unos valores de velocidad normal a la fibra de la madera, se han establecido dos intervalos para el diagnóstico de este tipo de madera:

- Velocidad normal (perpendicular a la fibra) $>1400 \mathrm{~m} / \mathrm{s}$ : madera sana.

- Velocidad normal (perpendicular a la fibra) $<1400 \mathrm{~m} / \mathrm{s}$ : madera con deterioro.

Asimismo a partir de las velocidades normales, obtenidas en una inspección in situ, se puede establecer, corrigiendo previamente estas velocidades para referirlas a una humedad del $12 \%$, la tensión básica de rotura de la probeta, [4] y [5]:

$$
\begin{aligned}
& \mathrm{f}_{\mathrm{k}}=\left(0,212 \mathrm{~V}_{\mathrm{N}}-183,09\right) \cdot 1 / 10 \quad\left(\mathrm{~N} / \mathrm{mm}^{2}\right) \\
& \mathrm{V}_{\mathrm{N} 12}=27,5\left(\mathrm{H}_{1}-12\right)+\mathrm{V}_{\mathrm{N} 1} \quad(\mathrm{~m} / \mathrm{s})
\end{aligned}
$$

Respecto a la técnica de la termografía de infrarrojos se trata de estudiar sus posibilidades de aplicación in situ como técnica no destructiva aplicada a un área importante y de difícil accesibilidad. Se pretende obtener, en una primera fase de inspección, datos necesarios para la evaluación del estado de la cubierta del edificio: contenido de humedad, zonas con pérdidas de densidad y deterioro, refuerzos y zonas de distintos materiales.

\subsection{Trabajos de laboratorio}

La metodología de ultrasonidos es el resultado de los trabajos realizados previamente en laboratorio donde a partir de los ensayos realizados a una serie de muestras se obtienen las rectas de regresión que relacionan las velocidades de transmisión ultrasónicas perpendiculares a la fibra con la tensión de rotura de la madera [4], [5].
Utilizando la técnica de la termografía, se ha realizado una batería de ensayos en laboratorio sobre madera de distintas especies (fundamentalmente pino silvestre/laricio) con el objetivo de determinar el alcance de la técnica controlando una serie de parámetros ambientales que, en circunstancias reales de aplicación, no sería posible. Estos estudios persiguen, entre otros, los siguientes objetivos:

- Determinar si es posible diferenciar distintas especies de madera en la medida que cada una de ellas presenta un valor de densidad distinto.

- Determinar el alcance de la termografía en la detección de defectos no superficiales, dependiendo del tamaño y la profundidad del mismo.

- Determinar el tamaño y profundidad de defectos detectables variando el contenido de humedad en los mismos.

También se estudia la posibilidad de detectar diferencias en las imágenes termográficas de una madera sana y una madera con deterioro y zonas con distintas humedades.

Sobre madera de la obra de San Felipe se han realizado ensayos de termografía de infrarrojos en restos del estribo de cubierta extraído debido a su mal estado causado por un ataque generalizado de pudrición parda. En estos ensayos se ha sumergido parte de la pieza en agua durante 2 horas y 30 minutos y se ha calentado en estufa durante 30 minutos a $80^{\circ} \mathrm{C}$. El objetivo es comprobar la diferencia térmica entre la zona humedecida y la zona seca. Los resultados y el termograma de este ensayo se muestran en apartados sucesivos.

\subsection{Trabajos de campo}

Los ensayos se han realizado en dos etapas: se realizó una primera visita en el mes de junio de 2009, cuando se estaba en fase de inspección de la cubierta y se habían desmontado algunos de los elementos de la cubrición, y en una segunda inspección en septiembre de 2009, cuando ya se habían realizado labores de tratamiento, sustitución y reparación de la estructura de madera.

Se han realizado ensayos de termografía de infrarrojos en todo el interior de la cubierta de madera accediendo al camaranchón que separa ésta de la bóveda encamonada. En esta zona se han tomado imágenes del conjunto de los pares y la tablazón, de los pares con signos externos de pudrición, de la zona con defectos (nudos y fendas) y de las zonas reparadas (en la $2^{\circ}$ visita). Las 
Tabla 1.

Condiciones ambientales de los días de realización de los ensayos.

\begin{tabular}{|c|c|c|c|c|}
\hline Día & Ta $^{\mathbf{a}}$ mínima & T $^{\text {a }}$ máxima & T $^{\text {a }}$ hora ensayo & Velocidad viento \\
\hline 9 junio & 18 & 21 & 20 & Sudoeste $(18 \mathrm{Km} / \mathrm{h})$ \\
\hline 24 septiembre & 20 & 27 & 23 & Este $(25 \mathrm{Km} / \mathrm{h})$ \\
\hline
\end{tabular}

condiciones ambientales durante las visitas realizadas se recogen en la Tabla 1. La hora de los ensayos de ambos días, 9 junio y 24 de septiembre, fue las 12 horas del mediodía. Cabe destacar que la velocidad del viento el día 24 de septiembre dificultó en gran medida la toma de imágenes. Los parámetros controlados directamente desde la cámara son la temperatura reflejada (no temperatura ambiente) y la emisividad de la madera.

Posteriormente, se han realizado ensayos de determinaciones de contenido de humedad y medidas de tiempos de transmisión de ondas ultrasónicas, mediante la metodología desarrollada por Rodríguez-Liñán C y Rubio De Hita, P. (1). La localización de los pares ensayados queda reflejada en rojo en los planos de planta (Figura 6).

La selección de los pares a ensayar se realiza tras una inspección visual y termográfica de la cubierta tomando las piezas que resultan más representativas según su nivel de deterioro o contenido de humedad (localizadas mediante la termografía), permitiendo extrapolar sus resultados al resto de la estructura. En cada uno de los pares se marcan tres secciones (a 0,5 m del empotramiento, a $1 \mathrm{~m}$ del empotramiento y a la mitad de la luz del par) y en cada sección se toman, análogamente, tres puntos de medida (en el centro de la sección y a $3 \mathrm{~cm}$ de los bordes superior e inferior). En cada uno de los puntos marcados se realizan medidas de transmisión ultrasónicas perpendiculares a la fibra obteniendo, finalmente, una velocidad de transmisión ultrasónica media para cada una de las secciones.

\section{RESULTADOS OBTENIDOS}

\subsection{De la termografía de infrarrojos}

De los termogramas realizados incluimos aquellos que tienen relevancia en el análisis que efectuamos:

Termograma 1. Vista del estribo tras el calentamiento realizado en laboratorio (Figura 7).

Termograma 2. Vista de conjunto del interior de la cubierta desde el camaranchón donde puede distinguirse la estructura de pares y la tablazón (Figura 8).

Termograma 3. Vista del par con deterioro superficial por pudrición parda (Figura 9).
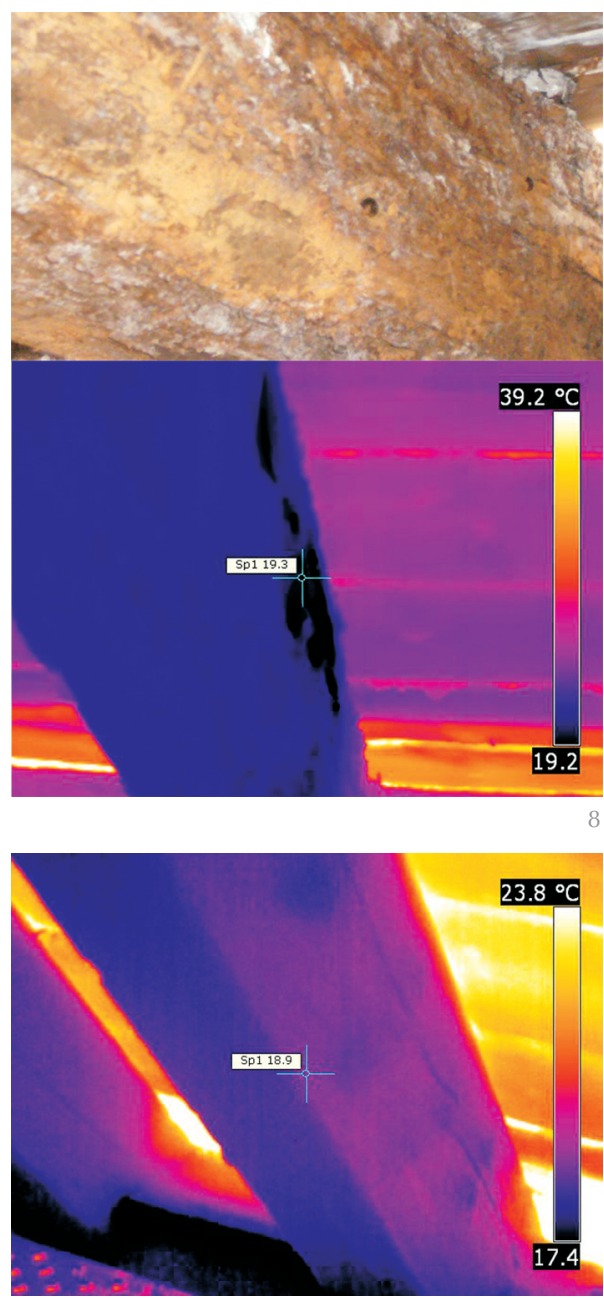
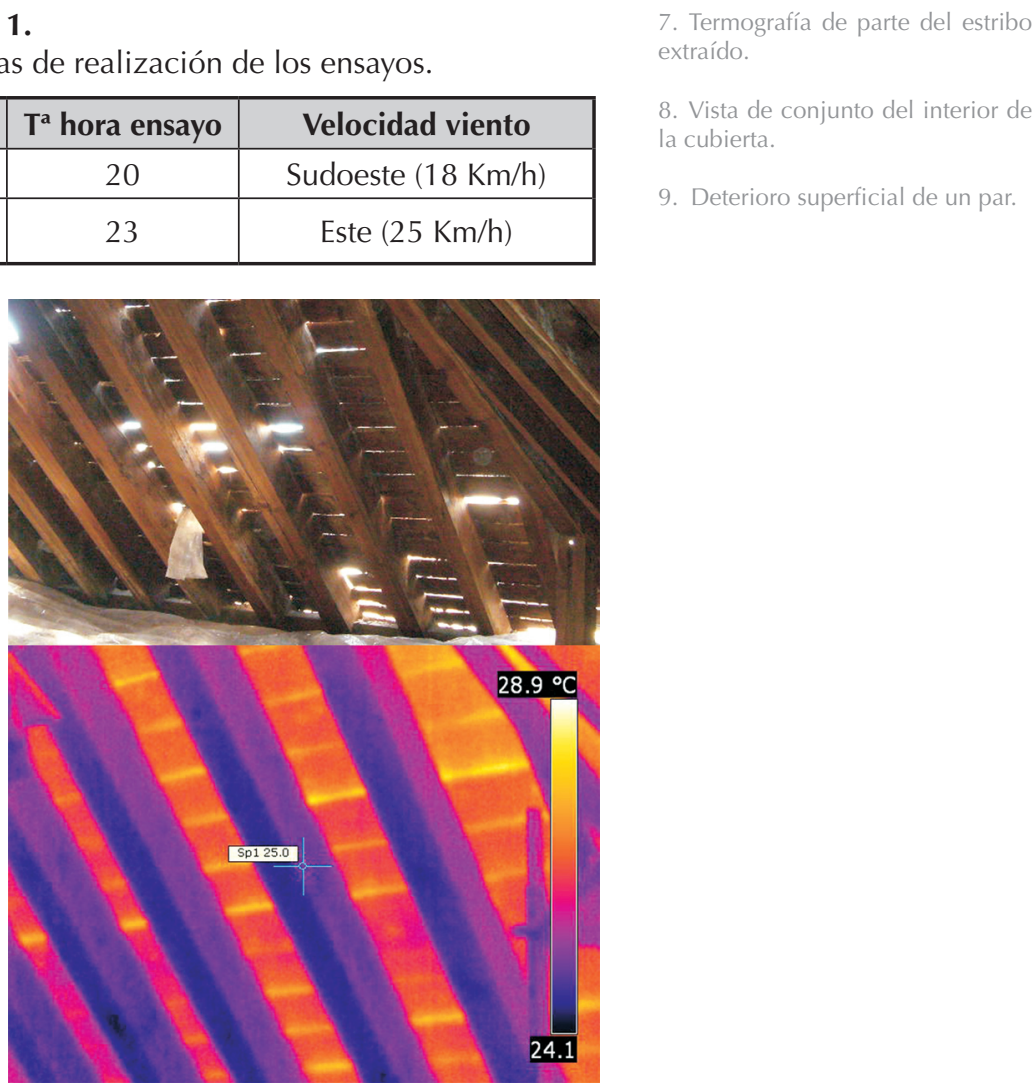
extraído.

8. Vista de conjunto del interior de la cubierta.

9. Deterioro superficial de un par. 
10. Fendas en un par.

11. Nudos en un par.

12. Empalme con madera nueva.

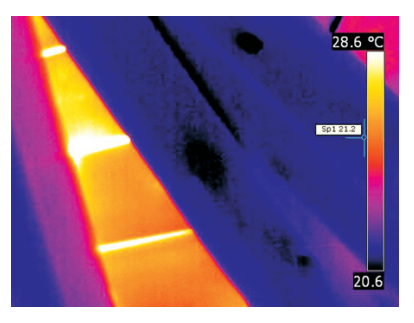

10
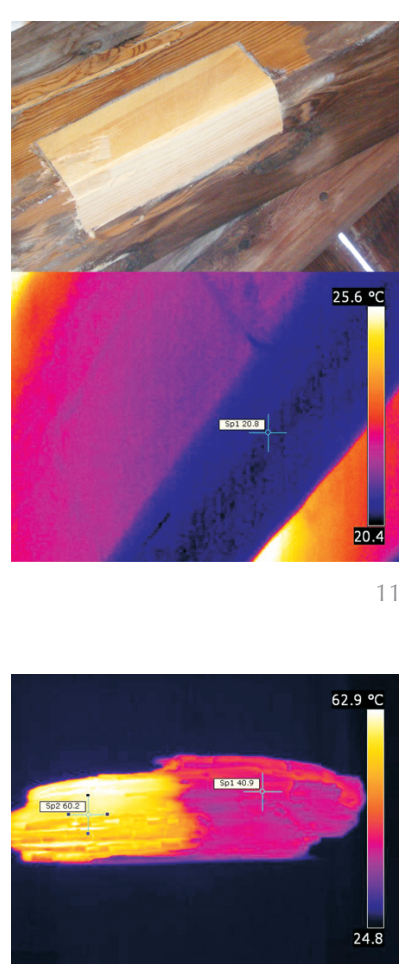

Termograma 4. Vista de un par con fenda (Figura 10).

Termograma 5. Vista de un par con nudos (Figura 11).

Termograma 6. Vista de par reparado con empalme de madera nueva (Figura 12).

\subsection{De los ensayos de ultrasonidos}

Las mediciones de los ensayos realizados (tiempo de ultrasonidos y determinaciones de humedad) en dos etapas distintas de la obra se recogen en la Tabla 2. Los ensayos en las secciones estudiadas se han realizado siguiendo la metodología descrita representando los valores medios de las secciones más representativas de la estructura. Los resultados de velocidad de ultrasonidos se refieren a una humedad del $12 \%$ por lo que los resultados obtenidos in situ (con humedades diferentes) de los diferentes elementos pueden ser comparados entre ellos aunque sus contenidos de humedad difieran.

\section{DISCUSIÓN DE RESULTADOS}

El uso conjunto de las técnicas de la termografía y de los ultrasonidos permite optimizar el rendimiento en la inspección de una estructura de madera y facilitar la labor de diagnóstico. Mediante una inspección inicial con termografía se detectan los focos de humedad y, por tanto, las zonas sensibles a presentar problemas de pudrición y pérdidas de densidad. Posteriormente, a través de los ultrasonidos, se cuantifica esa pérdida de densidad y resistencia. De esta forma se acotan rápidamente las zonas de estudio.

\subsection{De la termografía de infrarrojos}

De los ensayos de laboratorio se deduce que:
- Es posible detectar diferentes tipos de madera como resultado de una diferencia de densidad entre las mismas.

- Es posible detectar defectos por debajo de la superficie a partir de una diferencia térmica entre las distintas zonas. En estudios posteriores se detallarán las diferencias térmicas observadas en cada caso así como el rango de profundidad y tamaño de los defectos detectables. No obstante, puede subrayarse que, en todos los casos, es necesario aplicar una fuente de estimulación externa, lo que se conoce como termografía activa.

- La diferencia térmica entre las distintas zonas de ensayo se acentúa cuando el contenido de humedad no es uniforme.

Como método de análisis de la inspección in situ se ha realizado una comparativa entre la fotografía de un elemento o conjunto y su termografía (en el caso de no tener exactamente la misma toma se ha utilizado una similar). Las Figuras 7, 8 y 11 reúnen ambas vistas. Como resultado de este análisis, se puede establecer que la temperatura alcanzada por los distintos elementos de la cubierta y por lo tanto su imagen termográfica es distinta en:

1. Diferencia de humedad entre zonas (Figura 7).

La humedad, como la densidad o los defectos, es un parámetro que altera las propiedades térmicas de la madera. Esta diferencia de humedad se traduce en una diferencia térmica entre la zona humedecida (derecha de la imagen) y la zona seca (izquierda de la imagen) del estribo. El agua presente en la madera causa un incremento del calor específico, la conductividad y la densidad del material (10) lo que conlleva a que sea necesaria una mayor cantidad de energía para calentar la pieza.

Tabla 2

Resultados de ensayos de ultrasonidos.

\begin{tabular}{|c|c|c|c|c|}
\hline Zona & $\operatorname{Par} n^{\circ}$ & $\begin{array}{c}\text { Dimensiones } \\
(\mathrm{cm})\end{array}$ & $\begin{array}{l}\text { Vel. normal } \\
\text { media de ultra- } \\
\text { sonidos }(\mathrm{m} / \mathrm{s})\end{array}$ & $\begin{array}{l}\text { Humedad } \\
(\%)\end{array}$ \\
\hline $\begin{array}{c}\text { Sur } \\
\text { (junio 2009) }\end{array}$ & $\begin{array}{c}7 \\
\text { (pudrición parda) }\end{array}$ & $19,5 \times 28$ & 590,90 & 8,90 \\
\hline $\begin{array}{c}\text { Sur } \\
\text { (junio 2009) }\end{array}$ & $\begin{array}{c}8 \\
\text { (sano) } \\
\end{array}$ & $20 \times 25,5$ & $1.392,10$ & 9,30 \\
\hline $\begin{array}{c}\text { Sur } \\
\text { (septiembre 2009) }\end{array}$ & $\begin{array}{c}7 \\
\text { (reparado) }\end{array}$ & $19,5 \times 28$ & $1.251,80$ & 8,40 \\
\hline $\begin{array}{c}\text { Sur } \\
\text { (septiembre 2009) } \\
\end{array}$ & $\begin{array}{c}6 \\
\text { (sano) } \\
\end{array}$ & $20 \times 25$ & $1.647,30$ & 9,60 \\
\hline $\begin{array}{l}\text { Suroeste (altar mayor) } \\
\text { (septiembre 2009) }\end{array}$ & $\begin{array}{c}5 \\
\text { (sano, ligero ataque de car- } \\
\text { coma superficial común) }\end{array}$ & $19,5 \times 25$ & $1.149,80$ & 9,00 \\
\hline
\end{tabular}


2. La estructura de los pares y tablazón (Figura 8).

La temperatura superficial en elementos de madera viene dada por la temperatura del aire y por sus propiedades térmicas, calor específico, conductividad y difusividad, que dependen a su vez de la especie de madera, de la densidad o la humedad entre otros factores. Para el caso analizado, las condiciones exteriores del ensayo, temperatura del aire y humedad relativa, intervienen de igual manera sobre la temperatura superficial de los dos elementos. Así, puede deducirse que esta diferencia de temperaturas es el resultado de las propiedades térmicas de cada uno de los elementos de la estructura. El empleo de dos especies de maderas distintas, cada una de ellas con una densidad característica, y la diferencia de espesor entre las piezas se traducen en una diferencia térmica entre los elementos. El calor procedente del calentamiento solar de la cubierta es conducido a través de las piezas hasta alcanzar su cara opuesta que coincide con el interior de la estructura desde donde se toma la imagen termográfica. Así, como se revela en el termograma, la tablazón (piezas con menor espesor) alcanza una mayor temperatura interior que los pares en el mismo intervalo de tiempo adquiriendo a su vez un valor distinto de emisividad ( $T^{\mathrm{a}}$, longitud de onda).

3. Zonas con pérdida de densidad y elevada pudrición unido a un contenido de humedad superior (Figura 9).

Según Maldague (6) las propiedades térmicas de la madera sana y la madera deteriorada son distintas, como resultado de una diferencia de humedad entre ambas. En el caso de la madera deteriorada la presencia de humedad es mayor debido a que su estructura interna es menos densa. En esta imagen confirmamos la hipótesis en la que se fundamenta todo el trabajo de investigación de Maldague ya que existe una diferencia de temperatura entre la madera sana y la deteriorada resultado de esta diferencia de humedad y además, podemos añadir que si la zona donde se localiza el deterioro de la madera ya está seca (con el mismo contenido humedad que el resto de la madera) no se pueden apreciar diferencias térmicas notables mediante termografía aunque este deterioro sea superficial.

\section{Pares con fendas (Figura 10).}

La presencia de defectos en la madera interviene en la conductividad del calor provocando zonas de concentración o disipación de calor. En este caso, la apertura de la fenda provoca que el calor se disipe a través de la misma apareciendo reflejado en el termograma como una zona de menor temperatura. Por otro lado, este tipo de discontinuidades también pueden interpretarse como una diferencia de emisividad, ya que como vimos, este parámetro también depende del ángulo de incidencia que en el caso de la fenda varía.

En este caso cobra especial relevancia el tamaño del defecto (apertura de la fenda) y su relación con la distancia del ensayo y características de la cámara utilizada. Para este ensayo la imagen termográfica ha sido tomada a $1 \mathrm{~m}$ aproximadamente del defecto, pero este mismo defecto puede resultar imperceptible a mayores distancias.

\section{Pares con nudos (Figura 11).}

De forma análoga a las fendas, los nudos son defectos en la madera que provocan interferencias en el proceso de transmisión de calor. Se ha demostrado, mediante ensayos de laboratorio, que la temperatura superficial es función de la densidad. Así, los nudos son defectos con una densidad superior con respecto al resto de la madera sana, lo que conlleva a que sus propiedades térmicas (difusividad y conductividad térmicas) sean distintas y por tanto, alcancen una menor temperatura superficial, como queda reflejado en el termograma.

\section{Zonas reparadas con madera nueva}

(Figura 12).

En este caso una diferencia de densidad entre las distintas maderas conlleva a que sus propiedades térmicas sean diferentes y que puedan distinguirse en el termograma. Los ensayos realizados en laboratorio confirman que es posible distinguir especies de madera que tienen una densidad distinta.

\subsection{De los ensayos de ultrasonidos}

El análisis de los resultados nos muestra que para madera sin pérdida de densidad, (denominada madera sana en la Tabla $n^{\circ} 1$, ya que tan solo presenta un ligero ataque superficial en ciertas zonas de carcoma común), el rango de velocidades de ultrasonidos es bajo oscilando entre $1.149,80$ hasta $1.647,30 \mathrm{~m} / \mathrm{sg}$. con un valor medio de $1.360 \mathrm{~m} / \mathrm{sg}$. Esto nos indica que se trata de una madera de pino de baja densidad además con una gran cantidad de nudos de tamaño elevado que se detectan en la inspección visual. Por los estudios realizados (1) en maderas de similares características correspondería a una clase resistente C14 C16. En las zonas reparadas se ha empleado una madera de similares características.

Para la madera de aquellos pares, que en su encuentro con el estribo y en su cara superior tienen una pérdida de densidad 
elevada por un pudrición parda o cúbica avanzada, los valores de velocidad son muy inferiores, media de 590,9 m/sg, lo que confirma la capacidad de la técnica de ultrasonidos para detectar pérdidas de densidad en la madera estudiada. Estas zonas han sido sustituidas en el proceso de rehabilitación en curso utilizando una madera de similares características a la existente con uniones encoladas.

\section{CONCLUSIONES}

De todo este análisis podemos concluir que:

- La termografía de infrarrojos se revela como una técnica de inspección in situ de estructuras de madera, como grandes cubiertas, en espacios con una difícil accesibilidad.

- A partir de una inspección mediante termografía se puede conocer y aportar información sobre distintos aspectos: zonas con distinto contenido de humedad y que, por tanto, puedan presentar, en el momento de la inspección o en un futuro, signos de pudrición. También es posible distinguir maderas de distinta densidad (tablazón y pares y zonas con madera de reparación), zonas reparadas que presentan una discontinuidad del material, zonas con deterioro superficial (si éstas presentan un contenido de humedad superior a la madera sana) y pérdida generalizada por pudrición y la presencia de nudos y fendas.
- El viento atenúa las temperaturas superficiales de los cuerpos y las diferencias térmicas que pueda existir entre distintas zonas por el efecto de la convección enmascarando los resultados reales. Es por ello que las inspecciones termográficas deben realizarse en días sin viento. En nuestro caso, el día 24 de septiembre el viento cobró una especial relevancia y dificultó en gran medida la toma de imágenes.

- Para obtener datos más precisos sobre la pérdida de densidad por pudrición o ataque de xilófagos en el interior de la madera es necesario el uso de una técnica complementaria como pueden ser los ultrasonidos. Los ultrasonidos se presentan como una técnica eficaz para establecer un diagnóstico sobre el estado de deterioro de una estructura de madera permitiendo estimar su grado de deterioro.

Por lo tanto puede concluirse que la unión de una técnica de ultrasonidos y otra de termografía para la inspección in situ de estructuras de madera constituyen una buena herramienta para evaluar las condiciones de la estructura. La termografía permite detectar distintos contenidos de humedad y distintos materiales $y$, por otro lado, los ultrasonidos los distintos grados de deterioro o pérdida de densidad en zonas con elevado contenido de humedad de las piezas.

\section{BIBLIOGRAFÍA}

(1) Rodríguez Liñán C.; Rubio de Hita P.: "Evaluación del estado de la madera en obras de rehabilitación mediante técnicas de ultrasonidos". Universidad de Sevilla Secretariado de Publicaciones, 2000.

(2) Kaplan H.: "Practical applications of infrared thermal sensing and imaging equipment". SPIE Optical Engineering Press, Bellingam, Washington, 1999.

(3) Pitts D.; Sissom L.: "Heat transfer". Mc Graw Hill.

(4) Balageas, D.: "Termografía infrarroja: una técnica multifacética para la Evaluación No Destructiva (END)". IV Conferencia Panamericana de END, Buenos Aires, (2007).

(5) Wyckhuyse, X.; Maldague: "A study of Wood Inspection by Infrared Thermography, Part I: Wood Pole Inspection by Infrared Thermography". Research in Nondestructive Evaluation, Vol. 13, $\mathrm{n}^{\circ} 1$ (2001), pp. 1-12. DOI: 10.1007/s00164-001-0005-y.

Wyckhuyse, X.; Maldague: "Wood Inspection by Infrared Thermography, Part II", Research in Nondestructive Evaluation, Vol. 13, nº 1 (2001), pp. 13-21. DOI: 10.1007/s00164-001-0006-x.

(6) Tanaka, T.: "Wood inspection by thermography". 12th International Symposium on non-destructive testing of wood. Wood NTD-2000 Vol. 16, nº 03 (2001).

(7) Rodríguez Liñán C.; Rubio de Hita P.; Gómez de Cózar J.; Pérez Gálvez F.: "Diagnóstico mediante técnicas de ultrasonidos del forjado de madera del refectorio del Convento de Santa Clara en Carmona (Sevilla)". Informes de la Construcción, Vol. 55, nº 490 (2004), pp. 17-28.

(8) Pemán Medina, M.: "Contribución al estudio de la arquitectura gaditana. El maestro Afanador". Archivo Español de Arte, № 198, (Madrid, 1977), pp. 97-119.

(9) Pemán Medina, M.: "La Iglesia de San Felipe Neri, la arquitectura del templo y su significación a la luz de nuevos datos". Boletín del Museo de Cádiz, № 2, (Cádiz, 1980), pp. 29-100.

(10) Ludwig, N,; Redaelli, V.; Rosina, E.: Augelli, F.: "Moisture detection in wood and plaster by IR thermography", Infrared Physics \& Technology Vol. 46. (2004), pp. 161-166. 